\title{
A hypothetical cosmological test: Trigonometry on large scales
}

\author{
J. Liske \\ School of Physics and Astronomy, University of St Andrews, North Haugh, St Andrews KY16 9SS, UK \\ e-mail: j128@st-andrews.ac.uk \\ Received 8 August 2002 / Accepted 11 October 2002

\begin{abstract}
I discuss the constraints that could be placed on $\Omega_{\mathrm{M}}$ and $\Omega_{\Lambda}$ if it were possible to measure the redshift of an object at cosmological distance as observed by a second distant object. This hypothetical cosmological test has several attractive features. By a suitable choice of objects (in terms of distance from us and angular separation on the sky) a single measurement can constrain almost any given linear combination of $\Omega_{\mathrm{M}}$ and $\Omega_{\Lambda}$. These constraints do not depend on, or require marginalisation over, any other cosmological parameters (such as the Hubble constant) or any early universe physics. In principle, the test makes no assumptions about the objects involved and hence it is entirely independent of their physics and any possible evolution.
\end{abstract}

Key words. cosmological parameters

\section{Introduction}

Measuring cosmological parameters is a major industry and it has been argued persuasively that the era of quantitative (Efstathiou et al. 2002) or even precision (Schramm \& Turner 1998) cosmology has arrived. In particular, much attention has been devoted to the cosmological densities of mass, $\Omega_{\mathrm{M}}$, and vacuum energy, $\Omega_{\Lambda}$, because they determine the expansion history (and future) as well as the curvature of the universe. Over the decades, innumerable ways of measuring $\Omega_{\mathrm{M}}$ and/or $\Omega_{\Lambda}$ have been devised, ranging from the classical tests such as the magnitude-redshift (e.g. Riess et al. 1998; Perlmutter et al. 1999), the angular size-redshift (e.g. Kellermann 1993; Buchalter et al. 1998) or the number-magnitude (e.g. Phillipps et al. 2000) relations to their refinements such as the AlcockPaczyński test (Alcock \& Paczyński 1979) and the modern tests involving, e.g., the power spectrum of CMB fluctuations (e.g. de Bernardis et al. 2002), the clustering of galaxies (e.g. Peacock et al. 2001) or quasars (e.g. Hoyle et al. 2002) or the statistics of gravitational lensing (e.g. Fukugita et al. 1992).

Here, I discuss the constraints that could be placed on $\Omega_{M}$ and $\Omega_{\Lambda}$ if it were possible to measure (independently of cosmological parameters) the redshift of an object at cosmological distance as observed by a second distant object. In Liske (2000) (hereafter L00) I showed how to calculate this redshift from the redshifts and angular separation of the two objects as observed by us (see also Roukema 2001). The result depends only on $\Omega_{\mathrm{M}}$ and $\Omega_{\Lambda}$ and hence a comparison with measurements constrains these parameters.

The general idea is the following: if the curvature of the underlying manifold is known then two sides and an angle of a triangle fully determine the rest of the triangle. Conversely,

^ current e-mail: jol@roe.ac.uk three sides and an angle fully determine both the triangle and the underlying curvature. As usual, by expressing distances in terms of redshifts we make the connection to the expansion history of the manifold.

This idea combines many of the positive features of other cosmological tests while avoiding some of their problems. As pointed out above, $\Omega_{\mathrm{M}}$ and $\Omega_{\Lambda}$ are the only cosmological parameters involved. Any possible degeneracies with other parameters, such as the Hubble constant, $H_{0}$, are hence avoided. In principle, the test makes no assumption about the properties, the physics or the evolution of the objects involved. In particular, it does not require a standard candle or standard ruler. Hence there is also no restriction on the type of object. Any object observable over cosmological distances (e.g. galaxy, QSO, absorption line system) is, in principle, a potential candidate.

The main drawback of this test is that the measurement of a cosmological triangle's third side appears quite impossible! Just as we measure distances or redshifts between us and other objects exclusively from the photons received by them, we require as a prerequisite for the measurement of a triangle's third side the appearance of an object (in the widest possible sense) to depend on the radiation received from a another, specific object at cosmological distance. This is a rare scenario. For example, although the physics of galaxies is generally thought to depend on the cumulative radiation from other galaxies and quasars, i.e. the diffuse background, it is difficult to imagine any discernible difference in the appearance of, say, the Milky Way caused by the photons received from any other specific galaxy in the universe.

However, an effect may be observed in the vicinity of particularly luminous sources. For example, it is well known that the UV radiation of a QSO alters the ionization fraction of its surrounding intergalactic medium (the proximity effect, see 
e.g. Liske \& Williger 2001). Phillipps et al. (2002) pointed out that, potentially, this effect could be used to infer the luminosity distance between a QSO and a nearby absorber. In effect, this would be a measurement of the third side of a triangle. However, this measurement involves the intrinsic luminosity of the QSO which must be inferred from its apparent brightness and hence it is not independent of the metric.

Although no less useful, such tests, where the measurement of the third triangle side depends on the cosmological model, are less generic and conceptually different from the test proposed here. They depend on the details of the measurement of the third side and must be studied on a case-by-case basis, as was done by Phillipps et al. (2002) for the above example.

It appears then that the measurement of a cosmological triangle's third side is quite removed from observational reality. Hence the test must be considered as purely hypothetical, a thought experiment. Indeed, it is not even clear exactly which quantity might be "easiest" to measure (redshift, luminosity distance, etc.). Here, I have chosen to express the third side in terms of redshift, thus treating all three sides equally.

\section{Calculating $z_{2}^{\prime}$}

In the following I outline the method used to calculate the redshift of an object as seen by another, distant observer. I use the notation of L00 where I wrote the Robertson-Walker line element as

$$
\mathrm{d} s^{2}=-c^{2} \mathrm{~d} t^{2}+a^{2}(t)\left[\mathrm{d} \chi^{2}+\Sigma^{2}(\chi)\left(\mathrm{d} \theta^{2}+\sin ^{2} \theta \mathrm{d} \phi^{2}\right)\right] .
$$

The radial coordinate $\chi$ is dimensionless and $\Sigma(\chi)$ is defined as $\sin \chi, \chi$ or $\sinh \chi$ if $k=+1,0$ or -1 . Length dimensions are included in $a(t)$ by setting $a_{0}$ as the curvature radius for $k= \pm 1$ or $a_{0}=c H_{0}^{-1}$ for $k=0$.

Consider an object 1 observed by us today at $z_{1}$ and an object 2 at $z_{2}$ separated by an angle $\alpha$ on the sky (cf. Fig. 1 of L00). Object 2 emits a photon towards object 1 which is received by object 1 at the same time as object 1 emits the photon we receive from it today. I denote the redshift of object 2 as observed by object 1 as $z_{2}^{\prime}$ and the comoving coordinate distance between them as $\chi_{2}^{\prime}$. We have (L00)

$$
\begin{aligned}
\Sigma^{2}\left(\frac{\chi_{2}^{\prime}}{2}\right)= & \Sigma^{2}\left(\frac{\chi_{2}+\chi_{1}}{2}\right) \sin ^{2} \frac{\alpha}{2} \\
& +\Sigma^{2}\left(\frac{\chi_{2}-\chi_{1}}{2}\right) \cos ^{2} \frac{\alpha}{2}
\end{aligned}
$$

where $\chi_{1,2}=\chi\left(z_{1,2}\right)$. In L00 I set $\Omega_{\Lambda}=0$ and derived analytic expressions for the right and left-hand sides of Eq. (2) in terms of $z_{1}, z_{2}$ and $z_{2}^{\prime}$ respectively. In the case $\Omega_{\Lambda} \neq 0$ we relate the right-hand side of Eq. (2) to $z_{1}$ and $z_{2}$ by using

$$
\chi(z)=\sqrt{\left|\Omega_{k}\right|} \int_{0}^{z}[E(x)]^{-1 / 2} \mathrm{~d} x,
$$

where $\Omega_{k}=1-\Omega_{\mathrm{M}}-\Omega_{\Lambda}$ and

$$
E(z)=\Omega_{\Lambda}+(1+z)^{2} \Omega_{k}+(1+z)^{3} \Omega_{\mathrm{M}}
$$

When $\Omega_{k}=0$ then $\chi(z)$ is given by just the integral (i.e. we set $a_{0}=c H_{0}^{-1}$ ). We relate the left-hand side of Eq. (2) to $z_{2}^{\prime}$ by noting that an observer at $z_{1}$ would write the above as

$\chi_{z_{1}}(z)=\sqrt{\left|\Omega_{k}\left(z_{1}\right)\right|} \int_{0}^{z}\left[E_{z_{1}}(x)\right]^{-1 / 2} \mathrm{~d} x$,

where

$E_{z_{1}}(z)=\Omega_{\Lambda}\left(z_{1}\right)+(1+z)^{2} \Omega_{k}\left(z_{1}\right)+(1+z)^{3} \Omega_{\mathrm{M}}\left(z_{1}\right)$

and

$\Omega_{\Lambda}\left(z_{1}\right)=\frac{\Omega_{\Lambda}}{E\left(z_{1}\right)}$

$\Omega_{k}\left(z_{1}\right)=\frac{\left(1+z_{1}\right)^{2} \Omega_{k}}{E\left(z_{1}\right)}$

$\Omega_{\mathrm{M}}\left(z_{1}\right)=\frac{\left(1+z_{1}\right)^{3} \Omega_{\mathrm{M}}}{E\left(z_{1}\right)}$.

Hence we have $\chi_{2}^{\prime}=\chi_{z_{1}}\left(z_{2}^{\prime}\right)$. It is straightforward to show that

$\chi_{2}^{\prime}=\chi_{z_{1}}\left(z_{2}^{\prime}\right)=\chi\left(\tilde{z}_{2}\right)-\chi\left(z_{1}\right)$

where $\tilde{z}_{2}=\left(1+z_{1}\right)\left(1+z_{2}^{\prime}\right)-1$ is today's redshift of the photon emitted by object 2 and observed by object 1 to have a redshift of $z_{2}^{\prime}$. Finally, to calculate $z_{2}^{\prime}$ for a given $z_{1}, z_{2}$ and $\alpha$ we substitute Eq. (8) into Eq. (2) and numerically solve for $\tilde{z}_{2}$ using the Newton-Raphson method (Press et al. 2002), where the derivative of the function to be solved is simply given by

$\frac{\mathrm{d} \chi}{\mathrm{d} z}(z)=\sqrt{\frac{\left|\Omega_{k}\right|}{E(z)}}$.

\section{Constraints from a single $z_{2}^{\prime}$ measurement}

To investigate the potential of $z_{2}^{\prime}$ measurements for constraining $\Omega_{\mathrm{M}}$ and $\Omega_{\Lambda}$ I now use $\left(\Omega_{\mathrm{M}}, \Omega_{\Lambda}\right)=(0.3,0.7)$ as an input or reference cosmology in defining the quantity

$$
\begin{aligned}
& \chi^{2}\left(\Omega_{\mathrm{M}}, \Omega_{\Lambda}\right)= \\
& \quad \times \sum_{i=1}^{n_{p}}\left[\frac{z_{2}^{\prime}\left(0.3,07 ; z_{1}^{i}, z_{2}^{i}, \alpha^{i}\right)-z_{2}^{\prime}\left(\Omega_{\mathrm{M}}, \Omega_{\Lambda} ; z_{1}^{i}, z_{2}^{i}, \alpha^{i}\right)}{\sigma_{z_{2}^{\prime}}^{i}}\right]^{2}
\end{aligned}
$$

(not to be confused with the radial comoving coordinate). The sum goes over $n_{\mathrm{p}}$ pairs of objects, where the $i$ th pair is defined by $z_{1}^{i}, z_{2}^{i}$ and $\alpha^{i}$. $\sigma_{z_{2}^{\prime}}$ is the uncertainty in the measurement of $z_{2}^{\prime}$. In the absence of a specific notion of how a measurement of $z_{2}^{\prime}$ might be achieved, I consider $\sigma_{z_{2}^{\prime}}$ as an arbitrary scaling parameter for the moment.

First, let us consider $n_{\mathrm{p}}=1$, in which case $\chi^{2}$ simply reflects the variation of $z_{2}^{\prime}$ as a function of $\Omega_{\mathrm{M}}$ and $\Omega_{\Lambda}$. In Fig. 1 I plot $\chi^{2}\left(\Omega_{\mathrm{M}}, \Omega_{\Lambda}\right)$ for a number of different choices of $z_{1}, z_{2}$ and $\alpha$. Since one "data" point cannot constrain two free parameters, $\chi^{2}$ attains its minimum value not at a point but everywhere along a line in the $\Omega_{\mathrm{M}}-\Omega_{\Lambda}$ plane. I find that a single measurement of $z_{2}^{\prime}$ generally constrains a linear combination of the form $C=\Omega_{\Lambda}+m \Omega_{\mathrm{M}}$, although there are clearly exceptions to this rule, in particular at $z>2$ (cf. the last two panels 


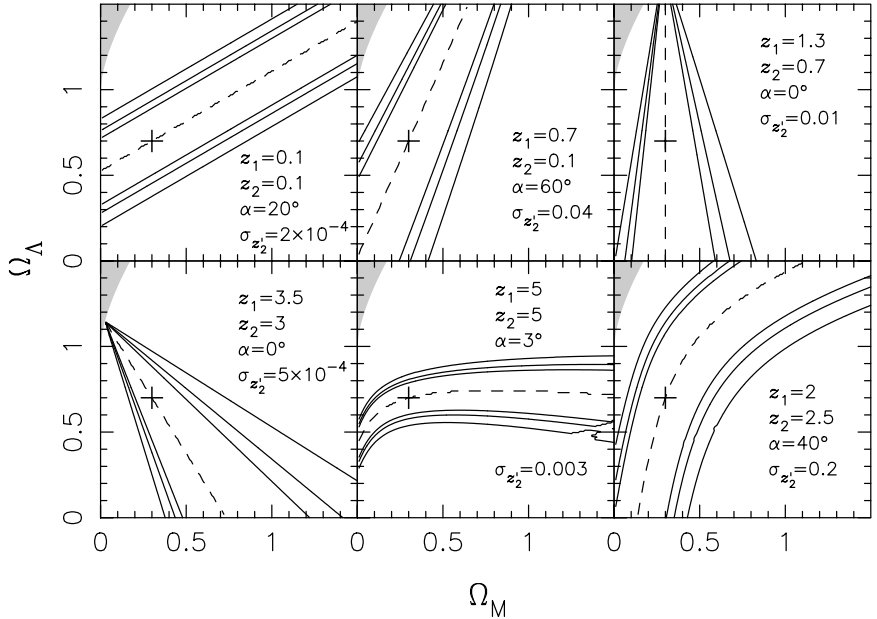

Fig. 1. Examples of the constraints placed on $\Omega_{\mathrm{M}}$ and $\Omega_{\Lambda}$ by a single measurement of $z_{2}^{\prime}$. Each panel shows a different choice of $z_{1}, z_{2}$ and $\alpha$. This figure demonstrates that, in principle, almost any given linear combination of $\Omega_{\mathrm{M}}$ and $\Omega_{\Lambda}$ can be constrained by a suitable choice of the cosmological triangle. The solid contours show the 68,90 and 99\% "confidence levels" (cf. Eq. (10)). In each panel, $\chi^{2}$ reaches its minimum everywhere along the dashed line, and $\sigma_{z_{2}^{\prime}}$, which scales the width of the contours, was chosen to give approximately similar widths in all panels. The cross marks the input cosmology of $\left(\Omega_{\mathrm{M}}, \Omega_{\Lambda}\right)=(0.3,0.7)$. The shaded region in the upper left corner of each panel represents "bouncing universe" cosmologies with no big bang in the past $(E(z) \leq 0$ at a finite $z>0$, see e.g. Carroll et al. 1992).

of Fig. 1). The examples in Fig. 1 further demonstrate that almost any value of $m$ is possible, including the cases $m=-\frac{1}{2}$, $m \rightarrow \infty, m=1$ and 0 , where the linear combination constrained is $C=-q_{0}$ (the deceleration parameter), $\Omega_{\mathrm{M}}, \Omega_{\text {total }}$ and $\Omega_{\Lambda}$ respectively.

In order to show the low-redshift characteristics of this constraint in more detail I have parameterised it in terms of the angle of the $\chi_{\min }^{2}$-line with the $\Omega_{\mathrm{M}^{-}}$axis, $\theta=\arctan (-m)$, and the formal error on $C, \sigma_{C}$, which is equivalent to the width of the contours in Fig. 1 (measured perpendicular to the $\chi_{\min }^{2}$ line). In Fig. 2 I plot $\theta$ and $\sigma_{C}$ for 284 different triangles with $0.1 \leq z_{1}, z_{2} \leq 1.5$ and $0^{\circ} \leq \alpha \leq 80^{\circ}$. Since $\sigma_{C}$ scales approximately linearly with the arbitrary value of $\sigma_{z_{2}^{\prime}}$ I plot the ratio of the two.

In the low-redshift limit, where all distances are small, the triangle cannot constrain the curvature but only $q_{0}$. This is a well-known property of many cosmological tests and due to the fact that, to second order, the expansion of the integral in Eq. (3) involves only $q_{0}$. For the redshift range shown, $\theta$ takes on all values from this low-redshift limit to $>90^{\circ}$ where $C=$ $\Omega_{\Lambda}$. In fact, the same value of $\theta$ can be produced by several, quite different configurations. For a given $z_{1}$, or if the values of $z_{1}$ and $z_{2}$ are exchanged, $\theta$ is generally larger for $z_{2}<z_{1}$, with the largest values achieved for $\alpha=0^{\circ}$.

Over the redshift range considered, $\sigma_{C}$ decreases by nearly four orders of magnitude. The increasing sensitivity to $\alpha$ with $z_{1}$ is due to a strong anti-correlation of $\sigma_{C}$ with $z_{2}^{\prime}$. The longer the third side of the triangle, the better the constraints on the cosmological parameters. This also explains why for a

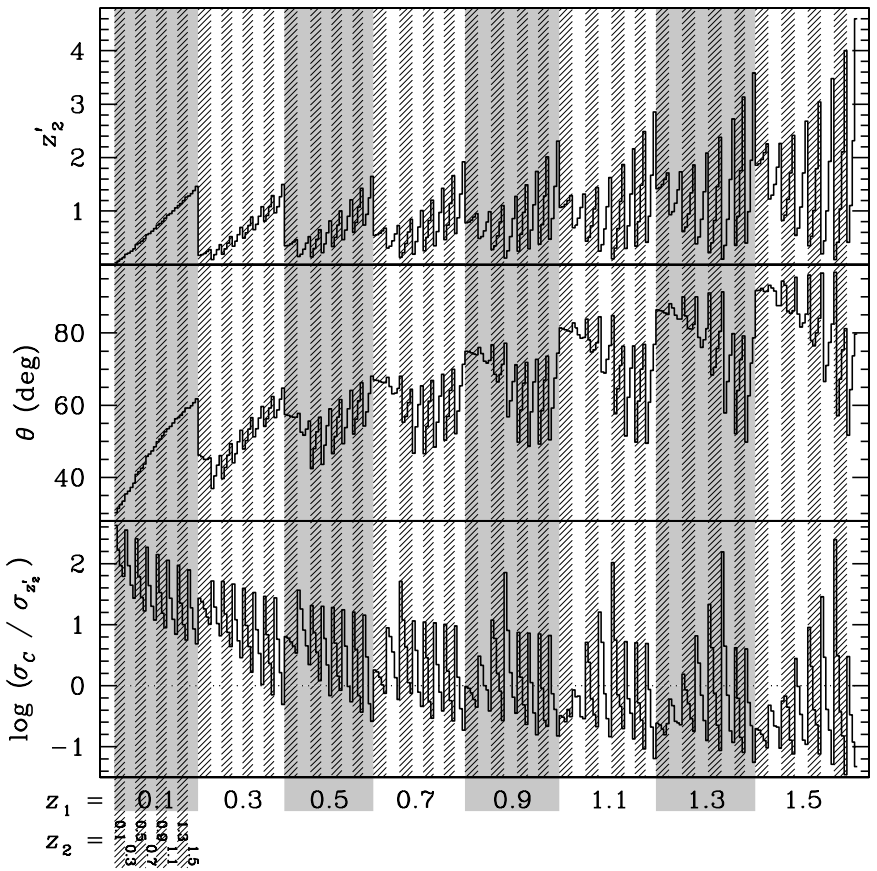

Fig. 2. Detailed behaviour of the constraint placed on the linear combination $C=\Omega_{\Lambda}+m \Omega_{\mathrm{M}}$ by a single $z_{2}^{\prime}$ measurement at $z \leq 1.5$. Each position along the $x$-axis corresponds to a different combination of $z_{1}, z_{2}$ and $\alpha$. Each change in background shading (grey and white) marks a change in the value of $z_{1}$ as indicated along the bottom axis. Similarly, for each value of $z_{1}$, hashed and non-hashed backgrounds mark the various values of $z_{2}$, which are also indicated along the bottom axis. Finally, for each combination of $z_{1}$ and $z_{2}, \alpha$ varies from $0^{\circ}$ to $80^{\circ}$. Constellations which do not constrain the cosmological model, i.e. $z_{1} \leq z_{2}$ and $\alpha=0^{\circ}$, have been excluded. There are no numerical errors or noise in this plot, the small-scale "spiky" behaviour is solely caused by the variation of $\alpha$. The top panel shows $z_{2}^{\prime}$. The middle panel shows $\theta=\arctan (-m)$. When $\theta=27^{\circ}, z_{2}^{\prime}$ constrains $C=-q_{0} ; \theta=90^{\circ}$ corresponds to a constraint on $C=\Omega_{\Lambda}$. The bottom panel shows the formal error on $C$ scaled by $\sigma_{z_{2}^{\prime}}$. This combination is approximately independent of the (arbitrary) value of $\sigma_{z_{2}^{\prime}}$.

given $z_{1}$ the tightest constraints are achieved for the largest $z_{2}$ and $\alpha$.

\section{Constraints from extragalactic redshift surveys}

The 2dF Galaxy and QSO Redshift Surveys (2dFGRS, Colless et al. 2001; 2QZ, Croom et al. 2001) are two of the largest redshift databases for the low and high redshift universes currently available. As an "application" of our thought experiment, consider the possibility that similar surveys have been carried out from the vantage point of another distant galaxy and that the results are sent to us. What would we be able to learn from these extragalactic redshift surveys?

In the following I consider two different scenarios with respect to the location of our "extragalactic collaborators" (object 1 in the terminology of Sect. 2). Both the 2dFGRS and 2QZ cover two distinct survey regions, near the North and South Galactic Poles (NGP and SGP), which lie approximately in opposite directions on the sky. First I will consider the case where object 1 lies in a direction roughly perpendicular to the 

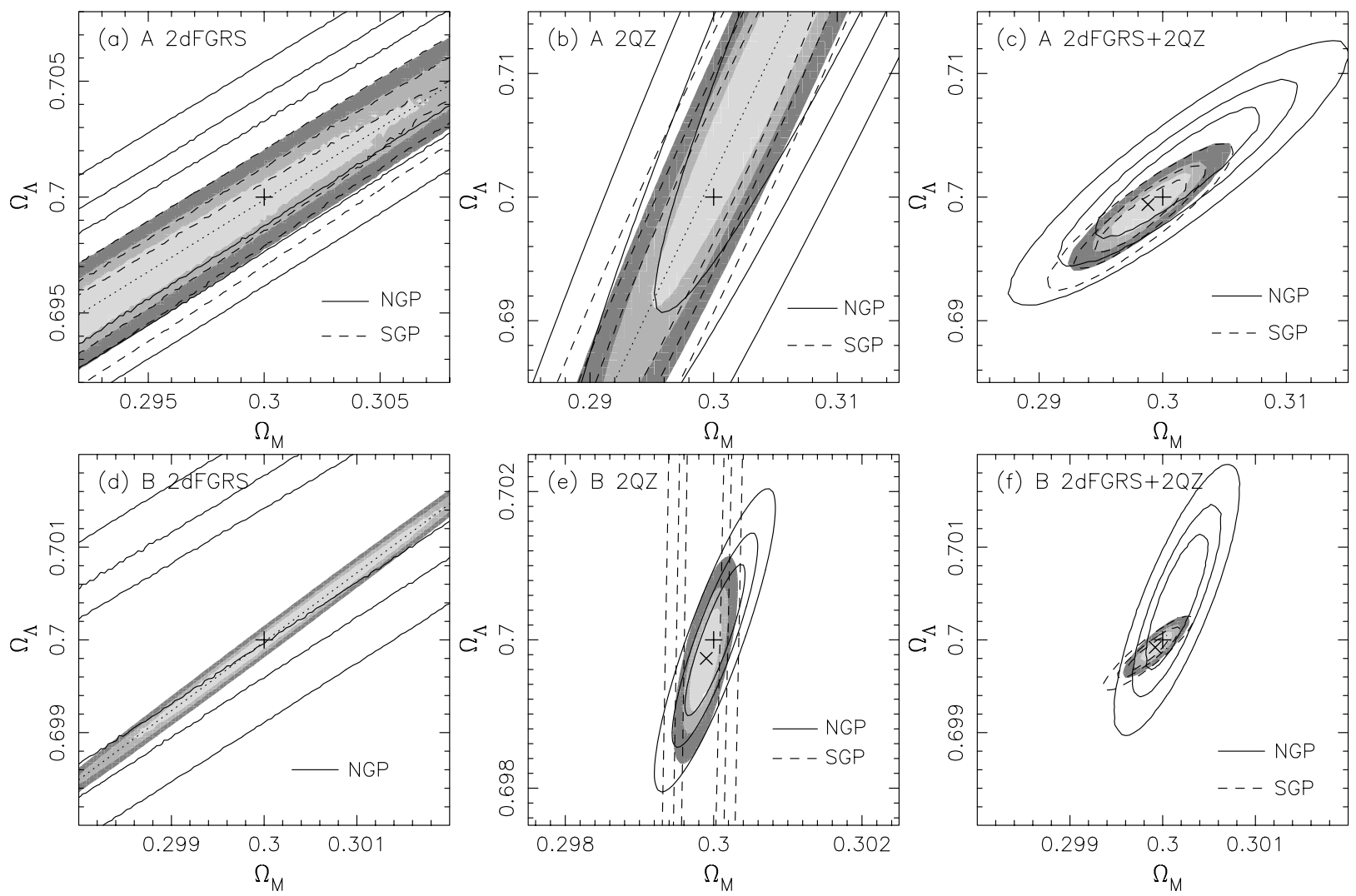

Fig. 3. Constraints on $\Omega_{\mathrm{M}}$ and $\Omega_{\Lambda}$ derived from combining the $2 \mathrm{dFGRS}$ and $2 \mathrm{QZ}$ with their extragalactic counterparts obtained from a distant galaxy (object 1). Each panel shows a different combination of case A/B (referring to the supposed location of object 1, see text) and redshift survey used. Each set of contours marks the 68, 90 and 99\% confidence levels. Solid contours show the constraints derived from the NGP alone, dashed contours refer to the SGP (omitted in panel d for clarity) and the greyscale contours show the joint NGP+SGP constraint. The "+" in each panel marks the input cosmology of $\left(\Omega_{\mathrm{M}}, \Omega_{\Lambda}\right)=(0.3,0.7)$ and the "X" in panels c), e) and f) shows the best fit. In panels a), b) and d) $\Omega_{\mathrm{M}}$ and $\Omega_{\Lambda}$ are not well constrained individually but only in a linear combination which is shown as the dotted lines.

NGP-Earth-SGP line and at a distance much smaller than the median redshifts of the $2 \mathrm{dFGRS}$ and $2 \mathrm{QZ}(\approx 0.1$ and 1.5 respectively). Specifically I choose $\alpha=06^{\mathrm{h}}, \delta=+80^{\circ}$ and $z_{1}=0.01$. In this case (which I will label case A) combining our own redshift surveys with those from object 1 affords us a stereoscopic view of these regions of the universe.

In the second scenario (case B) I take object 1 to be itself a representative member of the $2 \mathrm{dFGRS}$ or 2QZ. Specifically, in each case I choose a survey object near the centre of the NGP region and at the median redshift of the survey. For the 2dFGRS I choose object TGN182Z064 at $z_{1}=0.1$ and for the 2QZ I choose object J115952.8-012236 at $z_{1}=1.5$.

The datasets used in the following consist of the $100 \mathrm{~K}$ and $10 \mathrm{~K}$ public data releases of the 2dFGRS and 2QZ respectively. From the 2dFGRS I have selected all objects with quality $Q \geq 3$ and $0.01<z_{2}<0.5$ which resulted in 34489 and 53861 galaxies in the NGP and SGP respectively. From the 2QZ I have selected all objects classified as QSOs and with quality $q_{1}=11$, yielding 3965 and 6488 objects.

For case A I assume that all of the objects selected above have also been observed by object 1 , which implies that object 1's survey parameters are very similar to those of the actual 2dFGRS and 2QZ. For the case B/2dFGRS combination I make the same assumption, now implying that object 1's
Table 1. Summary of the constraints derived in Fig. 3.

\begin{tabular}{llr}
\hline \hline Case & Quantity constrained & Error $(99 \%)$ \\
\hline A 2dFGRS & $\Omega_{\Lambda}-0.62 \Omega_{\mathrm{M}}$ & $1.6 \times 10^{-3}$ \\
A 2QZ & $\Omega_{\Lambda}-2.08 \Omega_{\mathrm{M}}$ & $3.6 \times 10^{-3}$ \\
A 2dFGRS+2QZ & $\Omega_{\mathrm{M}}, \Omega_{\Lambda}$ & $5.3,4.0 \times 10^{-3}$ \\
B 2dFGRS & $\Omega_{\Lambda}-0.74 \Omega_{\mathrm{M}}$ & $1.2 \times 10^{-4}$ \\
B 2QZ & $\Omega_{\mathrm{M}}, \Omega_{\Lambda}$ & $3.4,7.9 \times 10^{-4}$ \\
B 2dFGRS+2QZ & $\Omega_{\mathrm{M}}, \Omega_{\Lambda}$ & $3.3,2.4 \times 10^{-4}$ \\
\hline
\end{tabular}

survey must be both wider and deeper. For the B/2QZ combination most of the SGP QSOs lie at very large redshifts as seen from object 1 and so I impose an upper limit of $z_{2}^{\prime}<5$, which leaves only 167 SGP QSOs in common to the 2QZ and its case B counterpart from object 1 .

In Fig. 3 I show $\chi^{2}\left(\Omega_{M}, \Omega_{\Lambda}\right)$ (see Eq. (10)) for each of the four combinations A,B/2dFGRS,2QZ as well as the joint constraints from combining the two surveys. Here, I have added Gaussian noise both to $z_{2}^{\prime}\left(\Omega_{\mathrm{M}}=0.3, \Omega_{\Lambda}=0.7\right)$ and $z_{2}$, assuming the same noise characteristics for $z_{2}^{\prime}$ as for $z_{2}$, i.e. for the 2dFGRS we have $\sigma_{z_{2}^{\prime}}=64,89,123 \mathrm{~km} \mathrm{~s}^{-1}$ for $Q=5,4,3$ (Colless et al. 2001) and for the 2QZ we have $\sigma_{z_{2}^{\prime}}=0.0035$ (Croom et al. 2001). 
In case A neither the $2 \mathrm{dFGRS}$ nor the $2 \mathrm{QZ}$ by themselves can constrain both $\Omega_{\mathrm{M}}$ and $\Omega_{\Lambda}$ individually (panels a and b). Both surveys only constrain a linear combination as in Sect. 3, shown as the dotted lines. However, since the linear coefficient $m$ is significantly different for the two surveys (cf. Table 1) using them jointly breaks the degeneracy and constrains $\Omega_{\mathrm{M}}$ and $\Omega_{\Lambda}$ to within $\sim 1 \%$ (panel c). Remarkably, it so happens that the 88350 low $-z$ objects of the 2dFGRS contribute almost equally to these constraints as the 10453 high- $z$ objects from the 2QZ. In this sense the two surveys are fortuitously well matched. The difference between the constraints from the NGP and SGP is purely due to the different numbers of objects in these regions.

For the B/2dFGRS combination (panel d) this difference is much larger. The reason is that in this case object 1 lies among the NGP galaxies which hence appear at much smaller $z_{2}^{\prime}$ than the SGP galaxies and thus give much weaker constraints. Again, only a linear combination of $\Omega_{M}$ and $\Omega_{\Lambda}$ is constrained. In contrast, the combination $\mathrm{B} / 2 \mathrm{QZ}$ does break the degeneracy (panel e). This is almost entirely due to the NGP QSOs, while the 167 SGP QSOs at $z_{2}^{\prime}<5$ contribute additional constraints on $\Omega_{\mathrm{M}}$ (but not $\Omega_{\Lambda}$ ). Jointly the two surveys constrain $\Omega_{\mathrm{M}}$ and $\Omega_{\Lambda}$ to within $\sim 0.1 \%$.

\section{Summary}

In this paper I have investigated the potential of trigonometry on cosmological scales for determining the cosmological parameters $\Omega_{\mathrm{M}}$ and $\Omega_{\Lambda}$. In particular, I have considered the possibility of measuring the redshift of an object at cosmological distance as seen by another distant object. I have shown that a single such measurement generally constrains a linear combination of the form $C=\Omega_{\Lambda}+m \Omega_{\mathrm{M}}$ and that $m$ can take almost any value depending on the constellation of the objects involved. Further I found that $\sigma_{C}$ decreases from $\sim 100 \sigma_{z_{2}^{\prime}}$ to $\sim 0.1 \sigma_{z_{2}^{\prime}}$ for $z_{1}=0.1$ to 1.5 . Finally I showed that the stereoscopic view afforded by combining the $2 \mathrm{dFGRS}$ and $2 \mathrm{QZ}$ with similar surveys undertaken from a galaxy at $z=0.01$ would enable us to constrain $\Omega_{\mathrm{M}}$ and $\Omega_{\Lambda}$ to within $\sim 1 \%$.

\section{References}

Alcock, C., \& Paczyński, B. 1979, Nature, 281, 358

Buchalter, A., Helfand, D. J., Becker, R. H., \& White, R. L. 1998, ApJ, 494, 503

Carroll, S. M., Press, W. H., \& Turner, E. L. 1992, ARA\&A, 30, 499

Colless, M., Dalton, G., Maddox, S., et al. 2001, MNRAS, 328, 1039

Croom, S. M., Smith, R. J., Boyle, B. J., et al. 2001, MNRAS, 322, L29

de Bernardis, P., Ade, P. A. R., Bock, J. J., et al. 2002, ApJ, 564, 559

Efstathiou, G., Moody, S., Peacock, J. A., et al. 2002, MNRAS, 330, L29

Fukugita, M., Futamase, T., Kasai, M., \& Turner, E. L. 1992, ApJ, 393, 3

Hoyle, F., Outram, P. J., Shanks, T., et al. 2002, MNRAS, 332, 311

Kellermann, K. I. 1993, Nature, 361, 134

Liske, J. 2000, MNRAS, 319, 557

Liske, J., \& Williger, G. M. 2001, MNRAS, 328, 653

Peacock, J. A., Cole, S., Norberg, P., et al. 2001, Nature, 410, 169

Perlmutter, S., Aldering, G., Goldhaber, G., et al. 1999, ApJ, 517, 565

Phillipps, S., Driver, S. P., Couch, W. J., et al. 2000, MNRAS, 319, 807

Phillipps, S., Horleston, N. J., \& White, A. C. 2002, MNRAS, 336, 587

Press, W. H., Teukolsky, S. A., Vetterling, W. T., \& Flannery, B. P. 2002, Numerical Recipes in C (Cambridge: Cambridge University Press)

Riess, A. G., Filippenko, A. V., Challis, P., et al. 1998, AJ, 116, 1009

Roukema, B. F. 2001, MNRAS, 325, 138

Schramm, D. N., \& Turner, M. S. 1998, Rev. Mod. Phys., 70, 303 